\section{How I diagnose and manage Philadelphia chromosome-like acute lymphoblastic leukemia}

\author{
Avraham Frisch ${ }^{1}$ and Yishai Ofran ${ }^{1,2}$ \\ ${ }^{1}$ Department of Hematology and Bone Marrow Transplantation, Rambam Health Care \\ Campus, Haifa, and ${ }^{2}$ Bruce Rappaport Faculty of Medicine, Technion, Israel Institute of \\ Technology, Haifa, Israel
}

\section{ABSTRACT}

A dvances in our understanding of mechanisms of leukemogenesis and driver mutations in acute lymphoblastic leukemia (ALL) lead to a more precise and informative sub-classification, mainly of B-cell ALL. In parallel, in recent years, novel agents have been approved for the therapy of B-cell ALL, and many others are in active clinical research. Among the newly recognized disease subtypes, Philadelphia-chromosomelike ALL is the most heterogeneous and thus, diagnostically challenging. Given that this subtype of B-cell ALL is associated with a poorer prognosis, improvement of available therapeutic approaches and protocols is a burning issue. Herein, we summarize, in a clinically relevant manner, up-to-date information regarding diagnostic strategies developed for the identification of patients with Philadelphia-chromosome-like ALL. Common therapeutic dilemmas, presented as several case scenarios, are also discussed. It is currently acceptable that patients with B-cell ALL, treated with an aim of cure, irrespective of their age, be evaluated for a Philadelphia-chromosome-like signature as early as possible. Following Philadelphia-chromosome-like recognition, a higher risk of resistance or relapse must be realized and treatment should be modified based on the patient's specific genetic driver and clinical features. However, while active targeted therapeutic options are limited, there is much more to do than just prescribe a matched inhibitor to the identified mutated driver genes. In this review, we present a comprehensive evidence-based approach to the diagnosis and management of Philadelphia-chromosome-like ALL at different time-points during the disease course.

\section{Introduction}

In recent years several new agents have been approved for the treatment of acute lymphoblastic leukemia (ALL), resulting in a tremendous improvement in longterm survival of patients. Concurrently, refinements in risk stratification have enabled escalation and de-escalation of therapy, thus minimizing treatment-related mortality, while maintaining high response rates. While the traditional method for subgrouping B-cell ALL (B-ALL) is based on cytogenetic and mutation analyses, it has been demonstrated that each of the known subgroups has a unique gene expression profile. Subsequent studies identified a B-ALL group which expresses the $B C R / A B L$ signature in the absence of the $B C R / A B L$ fusion, and hence this group was defined as Philadelphia chromosome-like (Ph-like) ALL.

Surprisingly, a search for genetic alterations driving these types of leukemia has revealed multiple mutations and/or aberrations, involving different signal transduction pathways. Clinically, patients with Ph-like ALL have been recognized as being at a high risk for a poor response to therapy or relapse. ${ }^{1.3}$ Herein we describe the challenges in the diagnosis and appropriate treatment selection for this heterogeneous group of patients.
Haematologica 2019
Volume 104(11):2135-2143

\section{Correspondence:}

YISHAI OFRAN

y_ofran@rambam.health.gov.il

Received: April 29, 2019.

Accepted: September 3, 2019.

Pre-published: October 3, 2019.

doi:10.3324/haematol.2018.207506

Check the online version for the most updated information on this article, online supplements, and information on authorship \& disclosures: www.haematologica.org/content/104/11/2135

(C)2019 Ferrata Storti Foundation

Material published in Haematologica is covered by copyright. All rights are reserved to the Ferrata Storti Foundation. Use of published material is allowed under the following terms and conditions:

https://creativecommons.org/licenses/by-nc/4.0/legalcode. Copies of published material are allowed for personal or internal use. Sharing published material for non-commercial purposes is subject to the following conditions: https://creativecommons.org/licenses/by-nc/4.0/legalcode, sect. 3. Reproducing and sharing published material for commercial purposes is not allowed without permission in writing from the publisher. 


\section{Driver mutations and aberrations in Philadelphia chromosome-like acute lymphoblastic leukemia}

In their landmark analysis of 1,725 ALL patients, Roberts et al. found kinase-activating mutations in more than $90 \%$ of patients with $\mathrm{Ph}$-like expression. ${ }^{4}$ The large variability of genetic alterations recognized in patients with Ph-like ALL makes further sub-categorization a challenge. For the purpose of a clinically oriented discussion, we believe clustering Ph-like ALL into the following four subgroups would be helpful.

\section{CRLF2-associated Philadelphia chromosome-like acute lymphoblastic leukemia}

The CRLF2 protein is a cytokine receptor which heterodimerizes with interleukin-7 receptor (IL7R)- $\alpha$, and upon binding to its ligand (thymic stromal lymphopoietin) activates the JAK-STAT pathway. This activation leads to cell proliferation without concomitant differentiation. ${ }^{5}$ In ALL, high expression of CRLF2 has been shown to correlate with reduced survival. ${ }^{4,6,7}$ Several genotypes are associated with high CRLF2 expression, including a chromosomal translocation with IGH-CRLF2 fusion, a cryptic interstitial deletion which results in a P2RY8CRLF2 fusion and CRLF2 point mutations engendering uncontrolled receptor activation.

The IGH-CRLF2 translocation is an early event in leukemogenesis and remains stable in relapse, while the P2RY8-CRLF2 translocation takes place later during disease development, is often subclonal and cannot be recognized in one-third to one-half of relapsed patients. ${ }^{8,9}$ Additionally, CRLF2 expression is 10-100-fold higher in patients with IGH-CRLF2 than in those with the P2RY8CRLF2.5,10,11 With regard to the prognostic impact, the relapse risk of IGH-CRLF2 ALL patients has been shown to be twice as high as that of P2RY8-CRLF2 ALL patients. ${ }^{12}$

Deregulation of CRLF2 expression is likely to require additional players to drive the leukemic process. In an ALL cell line with the IGH-CRLF2 translocation, knockdown of CRLF2 was not found to reduce proliferation of leukemic cells dramatically. ${ }^{5}$ About half of ALL patients with deregulated CRLF2 also have mutations in the JAKSTAT pathway ${ }^{4,7}$ and these latter are associated with a worse prognosis. ${ }^{4,13}$ In an analysis by the German Multicenter Study Group for Adult ALL (GMALL), onethird of adult patients with high CRLF2 expression were not found to harbor translocations or point mutations involving CRLF2. ${ }^{14}$ Similarly, in a recently published study, the CRLF2 translocation was identified in only $80 \%$ of Ph-like ALL patients demonstrating high CRLF2 expression. ${ }^{15}$ In fact, high CRLF2 immunophenotypic expression does not per se confer a worse prognosis, if it is not accompanied by CRLF2 genetic aberrations. ${ }^{11}$ Notably, high CRLF2 expression is reported to be significantly more frequent among patients of Hispanic ethnicity. 12,16

Mutations/deletions in the IKZF1 gene are prevalent in patients with Ph-like ALL ${ }^{1,17,18}$ and the presence of these mutations may be a better predictor of a poor prognosis than a high level of CRLF2 expression per se. ${ }^{17}$ Interestingly, a Chinese group recently demonstrated that IKZF1 is an epigenetic regulator of CRLF2, and IKZF1 mutations/deletions can lead to overexpression of CRLF2. ${ }^{19}$

\section{ABL-class translocations}

Translocations involving the pro-oncogenes $A B L 1$, ABL2, CSF1A and PGDFRB are evident in about $15 \%$ of Ph-like ALL cases. ${ }^{4,20}$ Due to the translocations, these genes lose their normal regulatory control; however, no specific partner genes, among the many reported, have been identified as being of particular prognostic significance. The presence of any of these translocations is considered sufficient for the diagnosis of Ph-like ALL. ${ }^{20}$ The translocations in question are mutually exclusive with CRLF2 and JAKSTAT mutations but, as in other Ph-like subgroups, are often concomitantly present with IKZF1 mutations/deletions. ${ }^{4,20}$ Patients with $A B L$-activating translocations usually respond poorly to therapy, continue to have measurable residual disease (MRD) after induction ${ }^{20}$ and should be treated with $A B L$ inhibitors, as discussed later.

\section{EPOR and JAK2 translocations}

$E P O R$ translocations, capable of partnering with multiple different genes, are grouped together with JAK2 translocations as they share the same mechanism of inducing cell proliferation through constitutive activation of the JAK pathway. These translocations are easy to recognize by fluorescence in situ hybridization (FISH) analysis and they are associated with a poor prognosis. ${ }^{4,21,22}$ EPORinvolving translocations lead to truncation of the erythropoietin receptor (EPO-R), its stabilization and overexpression, resulting in downstream activation of the JAK2 pathway. ${ }^{23}$ These chromosomal aberrations comprise about $10 \%$ of Ph-like ALL alterations, are associated with IKZF1 mutations or deletions and could potentially be targeted with JAK inhibition. ${ }^{17}$

\section{JAK/STAT or RAS mutations:}

This subgroup accounts for about $15-20 \%$ of Ph-like ALL cases. It includes genetic alterations of IL7R, FLT3, SH2B3, JAK1, JAK3, IL2RB and RAS genes. ${ }^{24}$ These mutations are all subclonal ${ }^{4}$ and there is paucity of data regarding the dynamics of their alterations in relapse. Remarkably, IKZF1 is less common in this subtype of $\mathrm{Ph}$ like ALL than in the above-mentioned ones. ${ }^{4,20,22}$ The prognosis of these patients is believed to be better than that of patients with other subtypes of Ph-like ALL., ${ }^{422}$ Individuals presenting with a RAS mutation as their sole driver mutation share both biological and clinical characteristics with the above-delineated JAK/STAT-derived group. Biologically, JAK/STAT and RAS signaling pathways are closely connected. Notably, other kinase mutations, i.e., NRAS, KRAS, PTPN11, and NF14 are observed not only in Ph-like ALL but also in hyperploid ALL. ${ }^{25,26}$ They are also found, with different prevalences, in all other subgroups of Ph-like ALL. 4,20,22

\section{Clinical presentation and diagnostic approaches to Philadelphia chromosome-like acute lymphoblastic leukemia}

The prevalence of Ph-like ALL in cohorts of newly diagnosed pediatric patients is about $10-20 \%,{ }^{1,3,4,18}$ and rises to $20-30 \%$ in adults. ${ }^{13,14,27} \mathrm{Ph}$-like cases are by definition $B C R / A B L$-negative, and are also always $M L L-$, ETV/RUNX1- and TCF3/PBX1-negative. Thus, they constitute a subgroup within the B cell other ALL. ${ }^{17,28}$ It has been previously reported that some patients may present 
with an overlapping group of hyperploid cytogenetics and Ph-like ALL. ${ }^{11,28}$ A recent comprehensive and integrative genomic classification of B-ALL categorized 23 leukemia subclasses, clearly defined by a specific genetic aberration, thus minimizing overlaps with the Ph-like phenotype. ${ }^{29}$

There is no consensus approach to the diagnosis of patients who express a Ph-like gene signature. ${ }^{30}$ These patients usually present with a higher white blood cell count $t^{1,4,17,22,27,31}$ and are likely to remain MRD-positive following standard induction regimens. , $13,14,22,28,31$ Selecting an optimal screening method and defining the patient population to be screened are still moving targets.

When first recognized, Ph-like ALL were retrospectively identified based upon gene expression profiling of a very wide array of genes. ${ }^{1,4,32}$ Notably, two large gene arrays, ${ }^{1,4}$ using a 257 -gene probe set and a 110-gene set ${ }^{2}$ shared only a minimal number of genes. Application of both arrays to each of two different cohorts of patients resulted in low concordance. ${ }^{2}$ Remarkably, kinase fusion cases in the two cohorts were identified by both methods in complete concordance, while there were many cases of high expression of CRLF2 and JAK/STAT mutations that were recognized with the 257-gene probe set and not with the 110-gene set. ${ }^{2}$ Thus, while the evaluation of newly diagnosed "Bcell other" ALL patients should include an attempt to identify the Ph-like phenotype, ${ }^{33}$ a definitive diagnosis should not rely on the gene expression phenotype but rather on the identification of a genetic aberration in the cell signaling related gene. RNA sequencing enables both identification of a Ph-like phenotype and comprehensive analysis of aberrant translocations. As this method is technically complicated and unavailable in most centers, a routine diagnosis of Ph-like ALL requires a combination of a simple screening test and an ultimate method to identify the culprit leukemia driver in each patient.

One screening approach is to search for a specific phenotype using limited sets of genes. ${ }^{34}$ Alternatively, a panel of FISH probes or polymerase chain reaction (PCR) tests covering the most common $A B L, J A K / E P O R$ and CRLF2 translocations can be employed as a screening tool. ${ }^{27,35}$ Low density microarrays (LDA), using a limited number of genes were first employed by Harvey et al. ${ }^{36}$ With an array of only 15 genes the tests were highly sensitive and specific ( $93 \%$ and $89 \%$, respectively) for the identification of Ph-like ALL. The concordance between this assay and the result of the original 257-gene set analysis was only $87 \%$, mainly due to over-diagnosis of cases of high CRLF2 expression by the LDA. Application of this method in high-risk pediatric ALL patients failed to detect mutations in about $15 \%$ of LDA-positive patients. ${ }^{15}$ Interestingly, the study identified nine patients with CRLF2 translocations who were LDA-negative, which translated into a false negative value of less than $1 \%$. Other LDA with fewer genes were developed by Heatley et al. ${ }^{18}$ and Roberts et al. ${ }^{37}$ To simplify this approach, Chiaretti et al. used quantitative real-time PCR to assess expression levels of ten genes and create a Ph-like ALL predictor. ${ }^{27}$ Expressionbased screening methods identify a phenotype and should be followed by a search for a targetable genotype, either by RNA sequencing, whole exome or targeted PCR panel sequencing, or by multiple-probe FISH analysis. As mentioned above, false negative results are rare; however, there are a substantial number of cases presenting with an overexpression signature but with no detectable driver genetic aberration. The actual risk and clinical implications in such cases are unknown.

It is also possible to screen for Ph-like ALL by searching directly for specific translocations and mutations. In a study conducted by the research group from the Munich Leukemia Laboratory in Germany ${ }^{38}$ screening by multiple FISH probes and targeted PCR (ABL1, ABL2, CSF1R, $P D G F R B$ along with quantitative PCR for CRLF2) successfully identified all patients who had a Ph-like gene expression profile according to the aforementioned 257-gene set. Another advantage of this method is the option of using quantitative PCR of the found driver mutation for MRD detection, although the negative predictive value of each aberration should be evaluated separately. Cooperative groups and leading centers around the world use different methods for the identification of Ph-like ALL. In Europe, some groups employ multiplex PCR or commercially available targeted RNA sequencing kits, while others use a FISH panel for primary screening. In the USA, the Children's Oncology Group (COG) uses LDA as the screening approach. Comprehensive RNA sequencing is conducted only in specific centers such as the St. Jude Medical Center.

The variability of the methods available makes the diagnosis of a Ph-like expression signature in a patient with no defined genetic alteration a challenge. Figure 1 presents a suggested clinical screening algorithm for Ph-like ALL to be applied outside of clinical trials.

\section{Treatment of Philadelphia chromosome-like acute Iymphoblastic leukemia}

To illustrate some of the key therapeutic issues and dilemmas in Ph-like ALL, we present and discuss several case scenarios. The discussion focuses on possible benefits of induction therapy intensification for these patients, post-induction treatment in MRD-positive and -negative patients as well as management of the most challenging cases of relapsed and elderly patients.

\section{Is there any preferred induction regimen for patients presenting with Philadelphia chromosome-like acute lymphoblastic leukemia?}

Case presentation. A 57-year old previously healthy man diagnosed with pre-B ALL has just been transferred from a rural hospital to your center. His peripheral blast count at diagnosis was $32 \times 10^{9}$ cells/L which dropped significantly after 1 week of steroid therapy. FISH panel analysis identified the EPOR translocation as the sole cytogenetic aberration. What are preferable induction therapy options?

Remission induction protocols commonly employed in ALL are variations of a consensus basic paradigm, combining four or five of the following drugs: anthracyclines, vincristine, cyclophosphamide, L/PEG-asparaginase and steroids. Differences between the protocols lie in their intensity, schedule and the addition of 6-mercaptopurine, cytarabine and rituximab. Data derived from randomized comparisons are scanty and inconclusive regarding survival superiority following any induction regimen, despite variations in remission rates in specific subgroups. ${ }^{39-41}$ In current clinical practice, appropriate treatment intensity and chemotherapy doses are usually determined based on the risk of adverse events and not on disease characteristics. Thus, a patient's advanced age, co-morbidities and/or fragility would lead most physicians to prescribe 


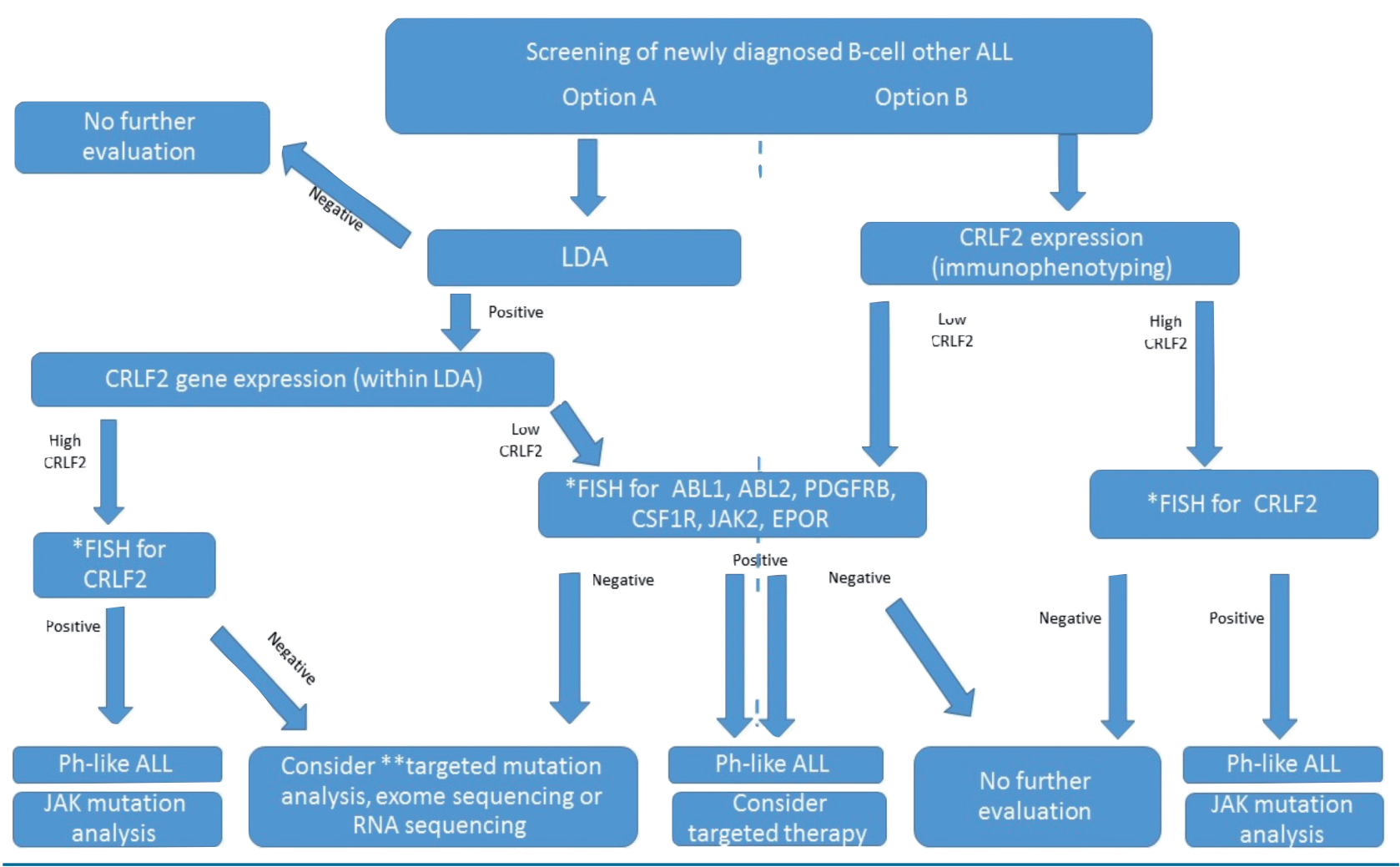

Figure 1. Screening of newly diagnosed cases of B-cell other acute lymphoblastic leukemia. *FISH-break-apart. **Targeted sequencing for mutations in JAK, IL7R, FLT3, SH2B3, RAS and PTPN11. ALL: acute lymphoblastic leukemia; LDA: low density microarray; FISH: fluorescence in situ hybridization; Ph-like: Philadelphia chromosome like.

low/moderate-intensity regimens, such as mini-HyperCVAD (cyclophosphamide and dexamethasone at $50 \%$ dose reduction, no anthracycline, methotrexate at $75 \%$ dose reduction, cytarabine at $0.5 \mathrm{~g} / \mathrm{m}^{2} \times 4$ doses) or other similar protocols. ${ }^{42-44}$

Until recently, the majority of Ph-like ALL patients were identified late during the course of treatment, usually after the completion of induction. Yet, with the implementation of CRFL2 immunophenotyping tests and routine application of wide-spectrum, rapid FISH panels and LDA, it is reasonable that a patient could be diagnosed with a Ph-like aberration early during induction. Clinical trials, such as the COG AALL1521, examining the benefit of adding ruxolitinib to standard induction, are currently recruiting patients. However, should the identification of an EPOR or JAK translocation entail alteration of the selected induction regimen for patients treated outside clinical trials? Ph-like ALL patients tend to remain MRDpositive after induction ${ }^{13,14}$ and are therefore planned for intensification of consolidation therapy by most pediatric protocols. ${ }^{45-47}$ Pediatric-oriented intensification regimens are extremely toxic and difficult to administer to high-risk adult patients. The presence of the EPOR translocation is an established adverse prognostic feature, ${ }^{5,23}$ but due to its rarity, randomized studies to assess a potential benefit of different induction or intensification regimens will probably never be conducted. In the absence of such clinical trials, intensified induction seems reasonable.

Among 148 recently reported adults with ALL, the achievement of MRD negativity did not translate into a better outcome in the 49 patients who were diagnosed with a Ph-like disease. ${ }^{13}$ These patients were treated with Hyper-CVAD or augmented BFM (Berlin-FrankfurtMunich) protocols with no specific intensification or modification for their high-risk ALL. In a pediatric series of 488 patients, those from the very high-risk group remained at a high risk of relapse even if MRD negativity was achieved. ${ }^{48}$ A new comprehensive study from the United Kingdom has suggested that the cutoff level for clinically relevant $M R D$ is different for various genetic subtypes of ALL. ${ }^{49}$ Thus, it is reasonable to consider all Ph-like ALL patients as high risk, regardless of their MRD status. Some data from 344 pediatric patients suggest that therapy intensification for Ph-like MRD-positive patients can lead to $\mathrm{MRD}$ eradication and improve outcome. ${ }^{28}$ However, confirmation from additional, large studies is required to feel confident about adopting chemotherapy intensification as a suitable therapeutic approach to be used as induction in Ph-like ALL patients. Notably, a randomized trial testing the value of enhancing therapy for MRD-positive patients, regardless of their genetic background, showed only a moderate improvement in event-free survival. However, even such benefit cannot be readily extrapolated to genetically very high-risk groups. ${ }^{50}$

As to practical suggestions for the patient in question, although not specifically tested in the context of Ph-like ALL, addition of rituximab if leukemic cells are CD20+ and the use of L-asparaginase or PEG-asparaginase, known to be active in high-risk ALL, may be recommended. At the same time, the risk of asparaginase-related complications at his age needs to be considered..$^{51-53}$

Blinatumomab, a bispecific antibody targeting CD19 
and CD3, has not yet been tested as an agent for Ph-like ALL treatment intensification. However, it has been proven to be effective in MRD eradication, and is currently being incorporated in clinical trials as part of front-line treatment for other high-risk ALL patients.

\section{Should targeted agents be added to induction regimens if the diagnosis of Philadelphia chromosome-like acute lymphoblastic leukemia is confirmed?}

Case presentation. A 23-year old woman with a recent diagnosis of $B$-ALL is being treated at your institution according to a GMALL protocol. ${ }^{54}$ On day 15 of induction, results of molecular tests reveal an IGH-CRLF2 translocation and a JAK2-activating mutation at R683G in the pseudo-kinase domain. Should JAK inhibitors be included in the treatment plan?

Most aberrations identified in patients with Ph-like ALL lead to kinase activation in the JAK2 pathway. ${ }^{55,56}$ Preclinical studies support the rationale that JAK inhibition would potentially counteract the aberrant, proliferation signal derived from the mutation..$^{57,58}$ Early-phase clinical trials have shown that the combination of JAK inhibitors with chemotherapy is safe and tolerable..$^{59-61}$ However, the clinical benefit of the addition of currently available JAK inhibitors, such as ruxolitinib, to chemotherapy in ALL is questionable. Unlike myeloproliferative neoplasms, in which JAK2 inhibition with low/intermediatedose ruxolitinib is sufficient to yield a clinical response, ${ }^{62}$ in leukemia, the use of ruxolitinib has not yet been approved. In addition, the proliferation signal in Ph-like ALL is derived from several kinases and parallel blockage of JAK $1 \& 2$, RAS and mTOR is probably needed for leukemia cell elimination. ${ }^{30,58,63-65}$ Thus, high ruxolitinib doses of at least $50 \mathrm{mg}$ twice daily ${ }^{60,66}$ could be required to achieve clinical benefit. Phase II studies exploring the role of incorporating ruxolitinib in induction regimens for $\mathrm{Ph}$ like ALL are ongoing.

For the minority of $\mathrm{Ph}$-like patients presenting with BCR-activating aberrations, data are accumulating that kinase inhibition by BCR/ABL specific tyrosine kinase inhibitors (TKI) may be beneficial. ${ }^{67-70}$ Most reports claiming the benefit of TKI present results of patients diagnosed with a PDGFRB translocation; so far, only a few cases of successful TKI use in patients with $A B L 1$ aberrations have been reported. ${ }^{4,71,72}$ Although no generalized conclusion can be made regarding the value of TKI in all ABL-activating cases, we feel that, given the established safety of these drugs and the data provided in the above-mentioned reports, arguments for off-label TKI use when considering targeted therapies for Ph-like ALL are much stronger than those for the use of JAK inhibitors. At the same time, one should bear in mind that Ph-like leukemia is a genetically complex disease and resistance to TKI, related to clonal evolution and appearance of additional mutations, has been reported..$^{73-75}$ Therefore, the addition of targeted therapy to first-line chemotherapy in Ph-like ALL is currently considered experimental.

\section{Approaches to post-remission therapy in patients with Philadelphia chromosome-like acute lymphoblastic leukemia with measurable residual disease}

Case presentation. A 63-year old man with B-ALL achieved complete remission after two cycles of Hyper-CVAD therapy. $M R D$ analysis by fluorescence activated cell sorting identified leukemic cells at a level of $4 \times 10^{-3}$ in the bone marrow. Results of molecular tests revealed the P2RY8-CRLF2 translocation. How should this patient be treated?

MRD monitoring is currently incorporated in ALL treatment protocols and decisions on the intensity of the firstline regimen and upfront allogeneic stem cell transplantation (SCT) rely mainly on the results of MRD evaluation. The likelihood of Ph-like ALL patients remaining MRDpositive after standard induction is high. MRD positivity at the end of induction is considered to be associated with a high risk of relapse in patients with Ph-like ALL as well as any other type of ALL. In such case, in adults, allogeneic SCT is strongly recommended, while some pediatric protocols would mandate intensification, not necessarily followed by allogeneic SCT. ${ }^{47,76}$ A retrospective study found no survival benefit from allogeneic SCT compared to chemotherapy intensification for high-risk pediatric patients. ${ }^{77}$ However, since results of allogeneic SCT are superior in patients who are MRD-negative prior to the transplant, ${ }^{78,79}$ intensification of therapy aiming to eradicate residual disease is logical even prior to allogeneic SCT. An analysis of the outcomes of 81 children treated in the ALL8 trial of the Australian and New Zealand Children's Haematology/Oncology Group (ANZCHOG) showed that even if considering only patients who achieved MRD negativity after allogeneic SCT, those who started conditioning with detectable MRD had a worse outcome. ${ }^{80}$ Although patients included in this study were not tested for the Ph-like signature, most of them were classified as "high-risk B-other" which probably overlaps with Ph-like ALL. A recent analysis of results of the ALL2008 study by the Nordic Society of Pediatric Hematology and Oncology (NOPHO) suggested that high-risk pediatric patients who remain MRD-positive at the end of consolidation will have a better outcome if residual disease is eradicated with intensive chemotherapy blocks prior to allogeneic SCT. ${ }^{81}$ Evidence supporting the administration of blinatumomab prior to transplant in an attempt to eliminate $\mathrm{MRD}$ is accumulating. Remarkably, based on a single-arm study, the Food and Drug Administration specifically approved the use of blinatumomab for high-risk B-ALL patients who achieve remission but remain $\mathrm{MRD}$-positive. In a prospective trial, $\mathrm{MRD}$ was eliminated in $78 \%$ of patients following blinatumomab treatment at a daily dose of $15 \mu \mathrm{g} / \mathrm{m}^{2}{ }^{82}$ The poor outcome of ALL patients older than 15 years, who remain $M R D$-positive after initial therapy and receive no blinatumomab prior to allogeneic SCT, was confirmed in a large European retrospective analysis. ${ }^{83}$ Apart from the recommendation of using this drug, additional treatment intensification may also be considered. For instance, idarubicin administration (for 3 days) just prior to busulfan/cyclophosphamide conditioning has been reported to improve post-SCT survival in MRD-positive patients. $^{84}$

As far as concerns the issue of MRD elimination in the Ph-like ALL setting, there are case reports demonstrating complete eradication or significant reduction of MRD at the time of allogeneic SCT resulting from pre-transplant intensification with ruxolitinib. ${ }^{61,85}$ However, the addition of targeted therapy, such as JAK or BCR/ABL inhibitors, should not substitute MRD eradication with blinatumomab or intensive chemotherapy prior to transplantation. Currently, no data are available to support maintenance with JAK2 inhibitors following allogeneic SCT in Ph-like ALL patients. While ruxolotinib has an immune 
suppressive effect and is suggested to be active against graft-versus-host disease, ${ }^{86,87}$ its routine use may prevent the benefit of the graft-versus-leukemia effect. For many years the association of graft-versus-host disease with graft-versus-leukemia activity has been considered questionable in ALL; yet, a currently published large retrospective study has confirmed an association between the presence of graft-versus-host disease and lower relapse rates. ${ }^{88}$ Moreover, a preclinical animal model challenges the effectiveness of ruxolitinib maintenance in prevention of leukemia relapse. ${ }^{89}$ Thus, ruxolitinib maintenance after allogeneic SCT should be considered experimental.

For patients with Ph-like ALL who carry an $A B L$-activating mutation the question of post-transplant TKI maintenance is unlikely to be answered in prospective clinical trials, mainly because of the rarity of this condition. However, safety data of post-transplant TKI maintenance can be extrapolated from the Philadelphia chromosome-positive ALL setting and encourage the use of this approach.

Persistence of MRD even after allogeneic SCT or failure to eradicate it prior to transplantation is a poor prognostic marker and a sign of impending relapse. In these circumstances, patients should be aggressively treated with intensive therapy individualized according to their treatment history and should be considered for chimeric antigen receptor T-cell therapy. Preclinical data suggest that for this very high-risk population, if IKZF1 mutations or deletions are present, a clinical trial with focal adhesion kinase (FAK) inhibitors could be an appropriate option..$^{90,91}$

\section{Patients with Philadelphia chromosome-like acute lymphoblastic leukemia who achieve measurable residual disease-negative remission}

Case presentation. A 42-year old woman presented with $B$ ALL with CRFL2 overexpression and a peripheral blood white blood cell count of $105 \times 10^{9} / \mathrm{L}$. Molecular evaluation identified the IGH-CRLF2 translocation. Following two cycles of Hyper$C V A D+P E G$-asparaginase she achieved molecular remission $\left[M R D\right.$-negativity $\left(<10^{-4}\right)$, not detected by multicolor flow cytometry assay]. How should this patient be further treated?

$\mathrm{MRD}$ is currently recognized as the most powerful risk factor in ALL patients. Absence of MRD, as evaluated by the tests capable of detecting even a low concentration $\left(10^{-4}\right)$ of leukemic cells, is associated with a superior outcome irrespective of molecular subtypes, patient's age and treatment protocols used..$^{77,92,93}$ However, in patients carrying high-risk molecular aberrations, such as Ph-like ALL patients, achievement of molecular remission does not completely abrogate the risk of a relapse. In the pediatric Australian ALL8 trial, among 666 recruited patients, the relapse risk was significantly higher in Ph-like patients with CRFL2 translocations than that in all non-Ph-like ALL patients $(57.8 \%$ vs. $16 \%$, respectively; $P<0.0001) .{ }^{80}$ Notably, ten of 14 (71.4\%) relapses in Ph-like ALL patients occurred despite the achievement of MRD negativity by day $79 .{ }^{18}$ Similar results were reported in the AIEOP-BFM ALL2000/R2006 study, in which a higher cumulative incidence of relapse was observed in Ph-like ALL patients (33.9\% vs. $14.9 \%$ in non-Ph-like ALL; $P=0.009$ ) even though a poor prednisone response and MRD positivity rates were identical in both groups. ${ }^{94}$ Similarly, data on adult patients demonstrated a high relapse rate in $\mathrm{Ph}$-like ALL patients, including those who achieved molecular remission, regardless of whether BFM-based or HyperCVAD regimens were used. ${ }^{13}$
A study by Roberts et al. suggested that risk-adapted therapy assigning patients with a high MRD level to allogeneic SCT could overcome the substantial risk of relapse. ${ }^{28}$ However, due to the lack of evidence supporting routine assignment to allogeneic SCT, a recent expert review and the updated recommendations from the European Working Group for Adult Acute Lymphoblastic Leukemia (EWALL) and the Acute Leukemia Working Party of the European Society for Blood and Marrow Transplantation (EBMT) advocated the use of allogeneic SCT during first complete remission only in MRD-positive pediatric and adult patients with Ph-like ALL. 95,96 Relapse rates in MRD-negative adults are higher than in pediatric patients with identical MRD kinetics of eradication. Therefore, in our opinion, a more liberal allogeneic SCT referral policy should be considered in adults with Ph-like ALL even if they achieve molecular remission. Additional risk factors, such as an IKZF1 alteration, have the potential to identify patients at the highest risk of relapse..$^{97-99}$ However, we are unaware of any available prospective data on patients' outcome following therapy stratification by IKZF1 alteration.

Relapse in patients with Philadelphia chromosome-like acute Iymphoblastic leukemia

Case presentation. A 7-year old child presented with B-ALL and an IGH-CRLF2 translocation. After COG-based induction, $M R D$ was detected at a level of $10^{-2}$ and high-risk intensive chemotherapy blocks were administered. Two weeks after the last chemotherapy the child had a full-blown hematologic relapse. How should this patient be managed?

Leukemia in patients presenting with early relapse right after intensive therapy is a devastating disease and prescribing additional chemotherapy seems futile. As described above, an anticipated effect of JAK inhibitors is modest and therefore in patients at a high risk of disease relapse immunotherapy should be the selected option. In CD19+ ALL, blinatumomab is an acceptable option for both adult $\mathrm{t}^{100}$ and pediatric patients. ${ }^{101,102}$ For adult patients, inotuzumab ozogamicin is also a valid option. ${ }^{103}$ Although still not widely available, chimeric antigen receptor $\mathrm{T}$-cell therapy is a powerful strategy to be used in such high-risk patients. A remission achieved with chimeric antigen receptor T-cell therapy should be followed by allogeneic SCT. ${ }^{104-106}$ To minimize the risk of CD19- escape and relapse, there is a rationale for combining CD19 with CD22-directed therapies and this combination should be evaluated against the risk of developing veno-occlusive disease during subsequent allogeneic SCT. ${ }^{107}$ Targeted therapy based on a patient's classification as having $\mathrm{Ph}$ like ALL and/or identification of a specific genetic aberration should not replace the use of other efficient agents available for the relapse setting.

\section{Patients of advanced age with Philadelphia chromosome-like acute lymphoblastic leukemia}

Case presentation. A 78-year old man who until recent days had been healthy with no chronic diseases, was admitted to hospital because of ALL. At presentation his white blood cell count was $55 \times 10^{\circ} / \mathrm{L}$, his hemoglobin concentration was $8.5 \mathrm{~g} / \mathrm{dL}$ and a spontaneous tumor lysis syndrome was diagnosed. Cytogenetic evaluation revealed an IGH-CRLF2 translocation and a molecular test identified an activating JAK2 mutation at the R683G position. How should this patient be treated?

Patients of advanced age diagnosed with ALL may 
achieve remission with intensive therapy but despite that are anticipated to experience a poorer survival mainly due disease relapse. ${ }^{108}$ The $\mathrm{Ph}$-like signature was reported in $24 \%$ of ALL patients over the age of $65^{22}$ but no prospective studies have included these patients, considering their genetic profile. Given that in most patients of advanced age, prolonged intensive chemotherapy followed by allogeneic SCT is not feasible, all such patients should be considered at high risk of relapse, regardless of their gene expression profile. The most promising approach thus far, which provided a considerable long-term survival in $\mathrm{Ph}$ negative ALL patients older than 60 years, was reported by Kantarjian et al. ${ }^{109}$ In their protocol, researchers from $\mathrm{MD}$ Anderson Cancer Center replaced a significant portion of chemotherapy with inotuzumab ozogamicin, hence creating a less toxic first-line regimen. ${ }^{109}$ With a median follow-up of 29 months, the 2-year progressionfree survival rate of 52 patients with a median age of 68 years was $59 \%$. Blinatumomab can also be safely added to such a protocol. ${ }^{110}$ We consider such a modified induction an acceptable approach for all Ph-negative ALL patients of advanced age. As previously discussed, the addition of targeted agents is rational only if $B C R / A B L$-activating genetic aberrations are identified and thus, for patients treated on such protocols molecular evaluation can be limited to $B C R / A B L$-activating lesions only. Outside of clinical trials, the patient in question should be treated with a less toxic regimen. Assuming that such regimen will not result in MRD eradication, blinatumomab should be added as early as possible, and inclusion of inotuzumab ozogamicin should be encouraged, if its off-label use is possible.

\section{Summary}

Patients with the $\mathrm{Ph}$-like gene expression pattern are at a high risk of relapse and theoretically could be offered treatment considering specific genetics of their disease. However, given that this group of patients is heterogeneous, it is unlikely that prospective studies will be conducted for each specific mutation to identify optimal treatment protocols. Moreover, no consensus exists regarding the preferred approach to be used for the diagnosis of $\mathrm{Ph}$ like ALL and management of a specific patient. Under these circumstances, the following three principles should guide the management of these patients. Screening for the Ph-like pattern should be adopted in routine practice in all patients. Patients should be informed that current screening methods may miss rare gene mutations that could be subject to off-label use of available targeted therapies (e.g., crizotinib); nevertheless, the effect of targeted therapy on such rare leukemic mutations has not been reported. If the ABL-activating aberration is identified, adding TKI to therapy is advised. All patients with identified kinase-activating aberrations should be defined as high risk; hence, intensification of chemotherapy, treatment with kinase targeting agents and/or antibody-derived novel agents may be considered.

\section{References}

1. Den Boer ML, van Slegtenhorst $M$, De Menezes RX, et al. A subtype of childhood acute lymphoblastic leukaemia with poor treatment outcome: a genome-wide classification study. Lancet Oncol. 2009;10(2):125134

2. Boer JM, Koenders JE, van der Holt B, et al. Expression profiling of adult acute lymphoblastic leukemia identifies a BCR-ABL1like subgroup characterized by high nonresponse and relapse rates. Haematologica. 2015;100(7):e261-264

3. Mullighan CG, Su X, Zhang J, et al. Deletion of IKZF1 and prognosis in acute lymphoblastic leukemia. $N$ Engl J Med. 2009;360(5):470-480

4. Roberts KG, Li Y, Payne-Turner D, et al. Targetable kinase-activating lesions in $\mathrm{Ph}$ like acute lymphoblastic leukemia. N Engl J Med. 2014;371(11):1005-1015.

5. Russell LJ, Capasso M, Vater I, et al. Deregulated expression of cytokine receptor gene, CRLF2, is involved in lymphoid transformation in B-cell precursor acute lymphoblastic leukemia. Blood. 2009;114(13): 2688-2698.

6. Yoda A, Yoda Y, Chiaretti S, et al. Functional screening identifies CRLF2 in precursor Bcell acute lymphoblastic leukemia. Proc Natl Acad Sci U S A 2010;107(1):252-257.

7. Chiaretti S, Brugnoletti F, Messina M, et al. CRLF2 overexpression identifies an unfavourable subgroup of adult B-cell precursor acute lymphoblastic leukemia lacking recurrent genetic abnormalities. Leuk Res. 2016;41:36-42.

8. Tsai AG, Yoda A, Weinstock DM, Lieber MR. $\quad t(X ; 14)(p 22 ; q 32) / t(Y ; 14)(p 11 ; q 32)$
CRLF2-IGH translocations from human Blineage ALLs involve CpG-type breaks at CRLF2, but CRLF2/P2RY8 intrachromosomal deletions do not. Blood. 2010;116(11): 1993-1994.

9. Vesely C, Frech C, Eckert C, et al. Genomic and transcriptional landscape of P2RY8CRLF2-positive childhood acute lymphoblastic leukemia. Leukemia. 2017;31(7): 1491-1501

10. Morak M, Attarbaschi A, Fischer S, et al. Small sizes and indolent evolutionary dynamics challenge the potential role of P2RY8-CRLF2-harboring clones as main relapse-driving force in childhood ALL. Blood. 2012;120(26):5134-5142.

11. Palmi C, Vendramini E, Silvestri D, et al. Poor prognosis for P2RY8-CRLF2 fusion but not for CRLF2 over-expression in children with intermediate risk B-cell precursor acute lymphoblastic leukemia. Leukemia. 2012;26(10):2245-2253

12. Ensor HM, Schwab C, Russell LJ, et al. Demographic, clinical, and outcome features of children with acute lymphoblastic leukemia and CRLF2 deregulation: results from the MRC ALL97 clinical trial. Blood. 2011;117(7):2129-2136

13. Jain N, Roberts KG, Jabbour E, et al. Ph-like acute lymphoblastic leukemia: a high-risk subtype in adults. Blood. 2017;129(5):572581.

14. Herold T, Schneider S, Metzeler KH, et al. Adults with Philadelphia chromosome-like acute lymphoblastic leukemia frequently have IGH-CRLF2 and JAK2 mutations, persistence of minimal residual disease and poor prognosis. Haematologica. 2017;102 (1):130-138.

15. Reshmi SC, Harvey RC, Roberts KG, et al.
Targetable kinase gene fusions in high-risk B-ALL: a study from the Children's Oncology Group. Blood. 2017;129(25):33523361

16. Konoplev S, Lu X, Konopleva M, et al. CRLF2-positive B-cell acute lymphoblastic leukemia in adult patients: a single-institution experience. Am J Clin Pathol. 2017;147 (4):357-363.

17. van der Veer A, Waanders E, Pieters $\mathrm{R}$, et al. Independent prognostic value of BCR-ABL1like signature and IKZF1 deletion, but not high CRLF2 expression, in children with Bcell precursor ALL. Blood. 2013;122(15): 2622-2629.

18. Heatley SL, Sadras T, Kok CH, et al. High prevalence of relapse in children with Philadelphia-like acute lymphoblastic leukemia despite risk-adapted treatment. Haematologica. 2017;102(12):e490-e493.

19. Ge Z, Gu Y, Zhao G, et al. High CRLF2 expression associates with IKZF1 dysfunction in adult acute lymphoblastic leukemia without CRLF2 rearrangement. Oncotarget. 2016;7(31):49722-49732.

20. Boer JM, Steeghs EM, Marchante JR, et al. Tyrosine kinase fusion genes in pediatric BCR-ABL1-like acute lymphoblastic leukemia. Oncotarget. 2017;8(3):4618-4628.

21. Jaso JM, Yin CC, Lu VW, et al. B acute lymphoblastic leukemia with $t(14 ; 19)$ (q32;p13.1) involving IGH/EPOR: a clinically aggressive subset of disease. Mod Pathol. 2014;27(3):382-389.

22. Roberts KG, Gu Z, Payne-Turner D, et al. High frequency and poor outcome of Philadelphia chromosome-like acute lymphoblastic leukemia in adults. J Clin Oncol. 2017;35(4):394-401.

23. Iacobucci I, Li Y, Roberts KG, et al. 
A. Frisch and Y. Ofran

Truncating erythropoietin receptor rearrangements in acute lymphoblastic

24. Roberts KG, Mullighan CG. Genomics in acute lymphoblastic leukaemia: insights and 2015;12(6):344-357.

25. Paulsson K, Horvat A, Strombeck B, et al. PTPN11 are frequent and possibly , and exclusive in high hyperdiploid childhood acute lymphoblastic leukemia. Genes
Chromosomes Cancer. 2008:47(1):26-33.

26. Wiemels JL, Rang M, Chang JS, et al. Backtracking RAS mutations in high hyper-
diploid childhood acute lymphoblastic leukemia. Blood Cells Mol Dis. 2010;45(3):

27. $186-191$

7. Chiaretti S, Messina M, Grammatico S, et al lymphoblastic lion of BCR/ABL1-like acute predictive statistical model based on quanttative real time-polymerase chain reaction
clinical, prognostic and therapeutic implicazions. Br J Haematol. 2018;181(5):642-652.

28. Roberts KG, Pei D, Campania D, et al.
Outcomes of children with BCR-ABL1-like acute lymphoblastic leukemia treated with risk-directed therapy based on the levels of
minimal residual disease. J Chin Oncol.

2014;32(27):3012-3020.
2. Gu Z, Churchman ML, Roberts KG, et al. PAX5-driven subtypes of B-progenitor acute lymphoblastic le

30. Ofran Y, Izraeli S. BCR-ABL (Ph)-like acute leukemia-Pathogenesis, diagnosis and hera-

peutic options. Blood Rev. 2017;31(2):11-16,
31. Harvey RC, Mulligan CG, Wang X, et al pediatric high-risk B-precursor acute lymphoblastic leukemia with gene expression
profiling: correlation with genome-wide profiling: correlation with genome-wide
DNA copy number alterations, clinical char2010;116(23):4874-4884.

32. Lo ML, Zhang J, Harvey RC, et al. Tyrosine
kinome sequencing of pediatric acute lymphoblastic leukemia: a report from the
Children's Oncology Group TARGET

Project. Blood. 2013;121(3):485-488.
33. Oran Y. Activated kinase in ALL: time to

34. Maese L, Tasian SK, Raetz EA. How is the
act. Blood 2017;29(25):328-328. Ph-like signature being incorporated into
ALL therapy? Best Bract Res Chin Haematol. ALL therapy? Best

35. Robin AJ, Peterson JF, Grignon JW Jr, et al. Identification of high-risk cryptic CRLF2
rearrangements in B-cell acute lymphoblas tic leukemia utilizing an FGFR3/IGH dualcolor dual-fusion DNA probe set. J Pediat
Hematol Oncol. 2017:39(4):e207-e210.

36. Harvey RC, Rang H, Roberts KG, et al. Development and validation of a highly sensitive and specific gene expression classifier
to prospectively screen and identify B-precursor acute lymphoblastic leukemia (ALL)
patients with a Philadelphia chromosomelike ("Ph-like" or "BCR-ABL1-like") signature
for therapeutic targeting and clinical interfor therapeutic targeting and clinic a
vention Blood. 2013;122(21):826.

37. Roberts KG, Reshmi SC, Harvey RC, et al. Genomic and outcome analyses of Ph-like
ALL in NCI standard-risk patients: a report from the Children's Oncology Group. Blood. 2018;132(8):815-824.

38. Fasan A, Kern W, Nadarajah N, et al. Three steps to the diagnosis of
Blood. 2015;126(23):2610.

39. Lamina N, Heffner LT, Kalaycio M, et al.
Treatment of adults with acute lymphoblastic leukemia: do the specifics of the regimen matter?: Results from a prospective random-

40. El-Cheikh J, El Dike I, Massoud R, et al. Hyper-CVAD compared with BFM-like acute lymphoblastic leukemia. A retrospective single-center analysis. Chin Lymphoma

41. Erkut N, Akidan O, Selim Batur D,
Karabacak V, Sonmez M. Comparison Karabacak V, Sonmez M. Comparison
between Hyper-CVAD and PETHEMA ALL-93 in adult acute lymphoblastic leukemia: a single-center study. Chemo-
therapy. 2018;63(4):207-213.

42. Garcia-Manero G, Kantarjian HM. The hyper-CVAD regimen in adult acute lym-
phocytic leukemia. Hematol Oncol Chin

43. Offidani M, Corvatta L, Malerba L, et al. Comparison of two regimens for the treat-
mont of elderly patients with acute lymphoblastic leukaemia (ALL). Leak
Lymphoma. 2005:46(2):233-238.

44. Sancho JM, Ribera JM, Xicoy B, et al. Results
of the PETHEMA ALL-96 trial in elderly of the PETHEMA ALL-96 trial in elderly negative acute lymphoblastic leukemia. Eur

45. Schmiegelow K, Forester E, Hellebostad M, et al. Long-term results of NOPHO ALL-92
and ALL-2000 studies of childhood acute lymphoblastic leukemia. Leukemia.
2010;24(2):345-354.

46. Moricke A, Zimmerman $\mathrm{M}$, Reiter $\mathrm{A}$, et al.
Long-term results of five consecutive trials in childhood acute lymphoblastic leukemia performed by the ALL-BFM study group
from 1981 to 2000. Leukemia.

2010;24(2):265-284.
47. Seibel NL, Steinherz PG, Gather HN, et al. Early postinduction intensification therapy
improves survival for children and addles improves survival for children and addlesOncology Group. Blood. 2008;111(5):2548-

48. Pui $\mathrm{CH}$, Pei D, Raimondi SC, et al. Clinical impact of minimal residual disease in chit-
dren with different subtypes of acute lymphoblastic leukemia treated with response-
adapted therapy. Leukemia. 2017;31(2):333-

49. O'Connor D, Enshaei A, Bertram J, et al. Genotype-specific minimal residual disease
interpretation improves stratification in pediatric acute lymphoblastic leukemia. J
Chin Oncol. 2018;36(1):34-43.

50. Vera A, Goulden N, Mitchell C, et al. Augmented post-remission therapy for a
minimal residual disease-defined high-risk subgroup of children and young people with
clinical standard-risk and intermediate-risk acute lymphoblastic leukaemia (UKALL 2003): a randomised controlled trial. Lancet
Oncol. 2014;15(8):809-818.

51. Maury S, Chevres S, Thomas X, et al. Rituximab in B-lineage adult acute lym-
phoblastic leukemia. N Angl J Med. 2016;

52. Cavell LA, Geller RD, Cohen HJ, et al. Four-agent induction and intensive asparagi-
nose therapy for treatment of childhood acute lymphoblastic leukemia. N Engr J

53. Fader S, Thomas DA, O'Brien $S$, et al.
Augmented hyper-CVAD based on doseAugmented hyper-CVAD based on doseasparaginase in adult acute lymphoblastic
leukemia salvage therapy. Chin Lymphoma
Myeloma Leuk. 2011;11(1):54-59.

54. Hoelzer D, Walewski J, Dohner H, et al.
Improved outcome of adult Burkitt lymphoma/leukemia with rituximab and chemotherapy: report of a large prospective
multicenter trial. Blood. 2014;124(26):3870-

55. Mulligh

JAK mutations in high-risk childhood acute U S A. 2009;106(23):9414-9418.

56. Harvey RC, Mullighan CG, Chen IM, et al.
Rearrangement of CRLF2 is associated with mutation of JAK kinase, alteration of IKZF1, Hispanic/Latino ethnicity, and a poor
outcome in pediatric B-progenitor acute lymphoblastic leukemia. Blood.

57. Maude SL, Tasian SK,

Targeting JAK1/2 and mOOR in murine phoblastic leukemia. Blood. 2012;120(17):

58. Tasian SK, Teachey DT, Li Y, et al. Potent efficacy of combined PI3K/mTOR and JAK els of Ph-like acute lymphoblastic leukemia.

59. Tasian SK, Assad A, Hunter DS, Du Y, Doh ML. A phase 2 study of ruxolitinib with
chemotherapy in children with Philadelphia chromosome-like acute lymphoblastic
leukemia (INCB18424-269/AALL1521): dose-finding results from the part 1 safety

60. Doh ML, Tasian SK, Rabin KR, et al. A phase 1 dosing study of ruxolitinib in children with
relapsed or refractory solid tumors, leukemics, or myeloproliferative neoplasms: A Children's Oncology Group phase 1 con-
sortium study (ADVL1011). Pediatr Blood Cancer. 2015;62(10):1717-1724.

al. Integration of ruxolitinib into Gale JM, et sifted therapy targeted against a novel JAK2 phoblastic leukemia. Pediatr Blood Cancer.

62. Bose P, Verstovsek S. JAK2 inhibitors for myeloproliferative neoplasm
Blood. 2017:130(2):115-125.

63. Nikolaev SI, Garieri M, Santoni F, et al. Frequent cases of RAS-mutated Down syn-
rome acute lymphoblastic leukaemia lack JAK2 mutations. Nat Commune. 2014;5:
4654 .

64. Suryani S, Bracken LS, Harvey RC, et al. Evaluation of the in vito and in vive efficacy
of the JAK inhibitor AZD1480 against JAKmutated acute lymphoblastic leukemia. Mol

65. Zhang Q, Shi C, Han L, et al. Inhibition of
mTORC1/C2 signaling improves antileukemia efficacy of JAK/STAT blockade in CRLF2 rearranged and/or JAK driven
Philadelphia chromosome-like acute B-cell lymphoblastic leukemia. Oncotarget.
2018;9(8):8027-8041. 66. Pemmaraju N, Kantarjian H, Nadia T, et al. A
phase I/II study of the Janus kinase (JAK)1 and 2 inhibitor ruxolitinib in patients with
relapsed or refractory acute myeloid leukemia. Chin Lymphoma Myeloma Leak. 67. Lengline E, Beldjord K, Dombret $\mathrm{H}$, et al. Successful tyrosine kinase inhibitor therapy
in a refractory B-cell precursor acute lymin a refractory B-cell precursor acute lym-
phoblastic leukemia with EBF1-PDGFRB
fusion. Haematological. 2013;98(11):e146148.

68. Weston BW, Hayden MA, Roberts KG, et al.

2142

haematological | 2019; 104(11) 
Tyrosine kinase inhibitor therapy induces remission in a patient with refractory EBF1PDGFRB-positive acute lymphoblastic leukemia. J Clin Oncol. 2013;31(25):e413416.

69. Kobayashi K, Miyagawa N, Mitsui K, et al. TKI dasatinib monotherapy for a patient with Ph-like ALL bearing ATF7IP/PDGFRB translocation. Pediatr Blood Cancer. 2015;62(6):1058-1060.

70. Zhang G, Zhang Y, Wu J, Chen Y, Ma Z. Acute lymphoblastic leukemia patient with variant ATF7IP/PDGFRB fusion and favorable response to tyrosine kinase inhibitor treatment: a case report. Am J Case Rep. 2017;18:1204-1208

71. Perwein T, Strehl S, Konig M, et al. Imatinibinduced long-term remission in a relapsed RCSD1-ABL1-positive acute lymphoblastic leukemia. Haematologica. 2016;101(8):e332335.

72. Frech M, Jehn LB, Stabla K, et al. Dasatinib and allogeneic stem cell transplantation enable sustained response in an elderly patient with RCSD1-ABL1-positive acute lymphoblastic leukemia. Haematologica. 2017;102(4):e160-e162.

73. Zhang $\mathrm{Y}$, Gao $\mathrm{Y}$, Zhang $\mathrm{H}$, et al. PDGFRB mutation and tyrosine kinase inhibitor resistance in Ph-like acute lymphoblastic leukemia. Blood. 2018;131(20):2256-2261.

74. Yeung DT, Moulton DJ, Heatley SL, et al. Relapse of BCR-ABL1-like ALL mediated by the ABL1 kinase domain mutation T315I following initial response to dasatinib treatment. Leukemia. 2015;29(1):230-232.

75. Zimmermannova O, Doktorova E, Stuchly J, et al. An activating mutation of GNB1 is associated with resistance to tyrosine kinase inhibitors in ETV6-ABL1-positive leukemia. Oncogene. 2017;36(43):5985-5994.

76. Stary J, Zimmermann M, Campbell M, et al. Intensive chemotherapy for childhood acute lymphoblastic leukemia: results of the randomized intercontinental trial ALL IC-BFM 2002. J Clin Oncol. 2014;32(3):174-184.

77. Conter V, Valsecchi MG, Parasole R, et al. Childhood high-risk acute lymphoblastic leukemia in first remission: results after chemotherapy or transplant from the AIEOP ALL 2000 study. Blood. 2014;123(10):14701478.

78. Knechtli CJ, Goulden NJ, Hancock JP, et al. Minimal residual disease status before allogeneic bone marrow transplantation is an important determinant of successful outcome for children and adolescents with acute lymphoblastic leukemia. Blood. 1998;92(11):4072-4079

79. Sramkova L, Muzikova K, Fronkova E, et al. Detectable minimal residual disease before allogeneic hematopoietic stem cell transplantation predicts extremely poor prognosis in children with acute lymphoblastic leukemia. Pediatr Blood Cancer. 2007;48 (1):93-100.

80. Sutton R, Shaw PJ, Venn NC, et al. Persistent $M R D$ before and after allogeneic BMT predicts relapse in children with acute lymphoblastic leukaemia. Br J Haematol. 2015;168(3):395-404.

81. Ifversen M, Turkiewicz D, Marquart HV, et al. Low burden of minimal residual disease prior to transplantation in children with very high risk acute lymphoblastic leukaemia: The NOPHO ALL2008 experience. Br J Haematol. 2019;184(6):982-993

82. Gokbuget N, Dombret H, Bonifacio M, et al. Blinatumomab for minimal residual disease in adults with B-cell precursor acute lym- phoblastic leukemia. Blood. 2018;131(14) 1522-1531

83. Gokbuget N, Dombret H, Giebel S, et al Minimal residual disease level predicts outcome in adults with Ph-negative B-precursor acute lymphoblastic leukemia. Hematology. 2019:24(1):337-348

84. Zhang $\mathrm{R}$, Lu $\mathrm{X}$, Wang $\mathrm{H}$, et al. Idarubicinintensified hematopoietic cell transplantation improves relapse and survival of highrisk acute leukemia patients with minimal residual disease. Biol Blood Marrow Transplant. 2019;25(1):47-55.

85. Ding YY, Stern JW, Jubelirer TF, et al. Clinical efficacy of ruxolitinib and chemotherapy in a child with Philadelphia chromosome-like acute lymphoblastic leukemia with GOLGA5-JAK2 fusion and induction failure. Haematologica. 2018;103(9):e427-e431.

86. Mori Y, Ikeda K, Inomata T, et al. Ruxolitinib treatment for GvHD in patients with myelofibrosis. Bone Marrow Transplant. 2016;51(12):1584-1587.

87. Zeiser R, Burchert A, Lengerke $C$, et al Ruxolitinib in corticosteroid-refractory graftversus-host disease after allogeneic stem cell transplantation: a multicenter survey. Leukemia. 2015;29(10):2062-2068.

88. Yeshurun M, Weisdorf D, Rowe JM, et al. The impact of the graft-versus-leukemia effect on survival in acute lymphoblastic leukemia. Blood Adv. 2019;3(4):670-680

89. Kim SK, Knight DA, Jones LR, et al. JAK2 is dispensable for maintenance of JAK2 mutant B-cell acute lymphoblastic leukemias. Genes Dev. 2018;32(11-12):849864

90. Churchman ML, Evans K, Richmond J, et al. Synergism of FAK and tyrosine kinase inhibition in Ph(+) B-ALL. JCI Insight. 2016;1(4).

91. Kurmasheva RT, Gorlick R, Kolb EA, et al. Initial testing of VS-4718, a novel inhibitor of focal adhesion kinase (FAK), against pediatric tumor models by the Pediatric Preclinical Testing Program. Pediatr Blood Cancer. 2017;64(4).

92. Conter V, Bartram CR, Valsecchi MG, et al. Molecular response to treatment redefines all prognostic factors in children and adolescents with B-cell precursor acute lymphoblastic leukemia: results in 3184 patients of the AIEOP-BFM ALI 2000 study. Blood. 2010;115(16):3206-3214.

93. Gokbuget N, Kneba M, Raff T, et al. Adult patients with acute lymphoblastic leukemia and molecular failure display a poor prognosis and are candidates for stem cell transplantation and targeted therapies. Blood. 2012;120(9):1868-1876

94. te Kronnie G, Silvestri D, Vendramini E, et al. Philadelphia-like signature in childhood acute lymphoblastic leukemia: the AIEOP experience. Blood. 2013;122(21):353.

95. Bhatt NS, Phelan R, Burke MJ. The role of hematopoietic stem-cell transplantation in first remission in pediatric acute lymphoblastic leukemia: a narrative review. Pediatr Rev. 2017;5(2):e10831

96. Giebel S, Marks DI, Boissel N, et al. Hematopoietic stem cell transplantation for adults with Philadelphia chromosome-negative acute lymphoblastic leukemia in firs remission: a position statement of the European Working Group for Adult Acute Lymphoblastic Leukemia (EWALL) and the Acute Leukemia Working Party of the European Society for Blood and Marrow Transplantation (EBMT). Bone Marrow Transplant. 2019:54(6):798-809.

97. Sutton R, Venn NC, Law T, et al. A risk score including microdeletions improves relapse prediction for standard and medium risk precursor B-cell acute lymphoblastic leukaemia in children. $\mathrm{Br} \mathrm{J}$ Haematol. 2018;180(4):550-562.

98. Olsson L, Ivanov Ofverholm I, NorenNystrom $U$, et al. The clinical impact of IKZF1 deletions in paediatric B-cell precursor acute lymphoblastic leukaemia is independent of minimal residual disease stratification in Nordic Society for Paediatric Haematology and Oncology treatment protocols used between 1992 and 2013. Br J Haematol. 2015;170(6):847-858.

99. Li JF, Dai YT, Lilljebjorn $\mathrm{H}$, et al. Transcriptional landscape of $B$ cell precurso acute lymphoblastic leukemia based on an international study of 1,223 cases. Proc Nat Acad Sci U S A. 2018;115(50):E11711E11720.

100. Kantariian H, Stein A, Gokbuget N, et al. Blinatumomab versus chemotherapy for advanced acute lymphoblastic leukemia. N Engl J Med. 2017;376(9):836-847.

101. Gore L, Locatelli F, Zugmaier G, et al. Survival after blinatumomab treatment in pediatric patients with relapsed/refractory B-cell precursor acute lymphoblastic leukemia. Blood Cancer J. 2018;8(9):80.

102.von Stackelberg A, Locatelli F, Zugmaier G, et al. Phase I/phase II study of blinatumomab in pediatric patients with relapsed/refractory acute lymphoblastic leukemia. J Clin Oncol. 2016;34(36):43814389

103. Kantariian HM, DeAngelo DJ, Stellies M, et al. Inotuzumab ozogamicin versus standard therapy for acute lymphoblastic leukemia. N Engl J Med. 2016;375(8):740-753.

104. Park JH, Riviere I, Gonen M, et al. Long-term follow-up of CD19 CAR therapy in acute lymphoblastic leukemia. N Engl J Med. 2018;378(5):449-459.

105. Maude SL, Frey N, Shaw PA, et al. Chimeric antigen receptor $\mathrm{T}$ cells for sustained remissions in leukemia. $\mathrm{N}$ Engl J Med. 2014;371(16):1507-1517

106. Maude SL, Laetsch TW, Buechner J, et al Tisagenlecleucel in children and young adults with B-cell lymphoblastic leukemia. N Engl J Med. 2018;378(5):439-448.

107. Jabbour EJ, Sasaki K, Ravandi F, et al. Inotuzumab ozogamicin in combination with low-intensity chemotherapy (miniHCVD) with or without blinatumomab versus standard intensive chemotherapy (HCVAD) as frontline therapy for older patients with Philadelphia chromosomenegative acute lymphoblastic leukemia: a propensity score analysis. Cancer. 2019;125 (15):2579-2586

108. Miller KC, Al-Kali A, Shah MV, et al. Elderly acute lymphoblastic leukemia: a Mayo Clinic study of 124 patients. Leuk Lymphoma. 2019;60(4):990-999.

109. Kantariian H, Ravandi F, Short NI, et al. Inotuzumab ozogamicin in combination with low-intensity chemotherapy for older patients with Philadelphia chromosomenegative acute lymphoblastic leukaemia: single-arm, phase 2 study. Lancet Oncol. 2018;19(2):240-248.

110. Jabbour E, Sasaki K, Ravandi F, et al. Chemoimmunotherapy with inotuzumab ozogamicin combined with mini-hyperCVD, with or without blinatumomab, is highly effective in patients with Philadelphia chromosome-negative acute lymphoblastic leukemia in first salvage. Cancer. 2018;124 (20):4044-4055. 